\section{Effects of Implementation Intentions on Anxiety, Perceived Proximity, and Motor Performance}

Personality and Social Psychology Bulletin 39(5) 623-635

(C) 2013 by the Society for Personality and Social Psychology, Inc Reprints and permission: sagepub.com/journalsPermissions.nav DOI: $10.1177 / 0146167213479612$ pspb.sagepub.com

\author{
Chadly Stern', Shana Cole', Peter M. Gollwitzer', \\ Gabriele Oettingen', and Emily Balcetis'
}

\begin{abstract}
Anxiety leads to exaggerated perceptions of distance, which may impair performance on a physical task. In two studies, we tested one strategy to reduce anxiety and induce perceived proximity to increase performance. We predicted implementation intentions that reduce anxiety would increase perceived visual proximity to goal-relevant targets, which would indirectly improve performance. In two studies, we induced performance anxiety on a physical task. Participants who formed implementation intentions to reduce anxiety perceived goal-relevant targets (e.g., golf hole, dartboard) as physically closer and performed better than both participants without a strategy (Study I) and participants with only a goal to regulate anxiety (Study 2). Furthermore, perceived proximity improved performance indirectly by increasing subjective task ease (Study 2 ). Results suggest that implementation intentions can reduce anxiety and lead to perceived proximity of goal-relevant targets, which helps perceivers make progress on goals.
\end{abstract}

\title{
Keywords
}

visual perception, implementation intentions, sports performance

Received January 6, 2012; revision accepted December 20, 2012

As Jean Van de Velde prepared to tee off for the final hole in the 1999 Open at Carnoustie, he seemed to be the guaranteed winner of the tournament. Unfortunately for Van de Velde, something went terribly wrong. His first shot landed far from his intended location, barely missing the water. His second shot bounced off of the grandstands, and his third landed in the sand pit. His score on that final hole lost him the lead and the championship. Before these successive shots, Van de Velde had golfed his finest throughout the tournament, which left spectators and commentators to wonder what thwarted his efforts to attain his goal.

One factor that contributes to performance failures is anxiety. For example, golfers under pressure, and most likely experiencing anxiety, found their putts landing farther away from a golf hole in comparison with golfers experiencing less pressure (Lewis \& Linder, 1997). Past research, primarily conducted on well-trained performers (Beilock \& Carr, 2001; Lewis \& Linder, 1997), suggests experienced performers "choke," or perform worse than expected, as feelings of pressure and anxiety mount. Like general arousal, anxiety is marked by increased physiological responses; however, anxiety in addition includes distinct cognitive components, such as intrusive, persistent, and ruminative negative thoughts tagged to specific eliciting events (Janelle, 2002; Martens, Burton, Vealy, Bump, \& Smith, 1990; Woodman \& Hardy, 2001). These types of anxious thoughts distract experienced performers from their reliance on procedural memory, which automatically engages practiced motor movements (Beilock \& Carr, 2001; Beilock \& Gray, 2007). Anxiety contributes to trained athletes' performance failures.

To be sure, research on choking among experienced performers bolsters the hypothesis that anxiety impairs performance. However, the mechanism by which this occurs may be different for inexperienced performers. In the present research, we focused on more general instances of physical action, where performers lack experience, expectations for performance standards, or procedural memory to enact motor movements. In such cases, anxiety may affect performance through different means; we chose to study visual perception. Specifically, we tested whether anxiety influences

\footnotetext{
'New York University, New York City, USA

Corresponding Author:

Chadly Stern, Department of Psychology, New York University, 6

Washington Place, New York, NY 10003, USA.

Email: cds330@nyu.edu
} 
visual perception, and in turn performance, for people who have not developed automatic procedural memories for physical performance.

The present research has three primary aims. First, this research explores the effects of anxiety on one component involved in performance- perceptions of distance to goalrelevant targets. Second, this research tests a strategy to reduce anxiety to influence distance perception. Third, this research explores whether and how perceptions of distance affect performance on a physical task. Overall, this work contributes to novel advances in testing the effects of a selfregulation strategy on anxiety reduction, perceived proximity, and performance, as well some mechanisms that contribute to these effects.

\section{Anxiety and Perception}

Anxiety influences the way people perceive the environment. Specifically, when people experience anxiety, aspects of the environment may appear more extreme. For example, when people are feeling more anxious, hills appear steeper (Stefanucci, Proffitt, Clore, \& Parekh, 2008) and the distance to the ground from a balcony ledge appears greater (Teachman, Stefanucci, Clerkin, Cody, \& Proffitt, 2008). Anxiety can exaggerate perceptions of the environment.

That anxiety can affect perception may be problematic during physical tasks when performers must use perceptual information to calibrate motor movements. First, if perceptions of the environment are distorted such that the environment appears exaggerated, people may perform worse because they calibrate movements erroneously. Second, exaggerated perceptions of distance may lead to subjective feelings that the task is more difficult, which could compound anxiety, lead to distracting thoughts, and impair performance. These two possibilities lead to the hypothesis that anxiety may exaggerate perceptions of the environment, which in turn may contribute to poor performance.

\section{Strategies to Mitigate Anxiety and Improve Performance}

Although it may seem an effective way of improving performance, reducing anxiety is complicated and often unsuccessful. Even strong desires to achieve performance goals are often insufficient to ward off unwanted anxiety (Baumeister \& Showers, 1986). However, one strategy that effectively mitigates anxiety and other negative internal states is the use of implementation intentions (Achtziger, Gollwitzer, \& Sheeran, 2008). Implementation intentions are if-then statements (e.g., "If situation $\mathrm{X}$ arises, then I will do Y!"). They specify a situational cue as the "if," along with a goal-directed response as the "then" (Gollwitzer, 1993; 1999). For example, a golfer who experiences anxiety that impairs his or her performance could form the implementation intention, "If I feel anxious, then I will tell myself to breathe deeply!" They are effective at regulating negative internal states, including anxiety, and help to block those states from interfering with performance (Achtziger et al., 2008; Schweiger Gallo, Keil, McCulloch, Rockstroh, \& Gollwitzer, 2009).

Implementation intentions are unique regulation strategies that differ from other means to mitigating negative affective states (i.e., "response-focused" strategies; Gross, 2001). Implementation intentions do not require extensive cognitive resources (Gollwitzer \& Sheeran, 2006), whereas emotion suppression strategies in which individuals consciously inhibit emotions require effort and are cognitively taxing (Gross, 2001; Richards \& Gross, 1999). In addition, implementation intentions allow participants to regulate anxiety while remaining committed to and engaged in the task. Because implementation intentions automatically activate goal-directed responses when situational cues arise, individuals can focus on tasks without diverting resources to cope with emotions (Bayer \& Gollwitzer, 2007). On the other hand, conscious emotion regulation strategies, like reappraisal (Gross \& Levenson, 1997), ask participants to adopt a detached perspective and withdraw from the task, which may inadvertently impair performance. Thus, implementation intentions differ from other emotion suppression strategies and may be more effective strategies for reducing anxiety during motor performance tasks.

\section{Emotion Regulation Strategies and Perception}

If negative affective states influence perception and if these states can impair performance, we argue that strategies to attenuate anxiety may indirectly lead to improved performance. Some research supports the first part of our proposition - that strategies to regulate anxiety do affect perception of the environment - but the evidence to date is inconclusive. When anxiety is high rather than low, individuals perceive their environment in more extreme ways; however, when strategies are used to reduce the anxiety, the perceptual distortions attenuate (see Rachman \& Cuk, 1992). Moreover, when researchers provided participants with instructions meant to increase the arousal they experienced while viewing graphic images, participants' perceptual distortions were amplified compared with a control condition (Stefanucci \& Storbeck, 2009). Attenuating and accentuating arousal and anxiety may impact perception.

However, not all regulation strategies are necessarily capable of impacting anxiety and perception. For example, when participants engaged in reappraisal by taking a "detached, third person perspective" when viewing gruesome pictures, neither their affective experiences nor subsequent perceptual estimates differed from those of participants in a control condition (Stefanucci \& Storbeck, 2009, Studies $4 \mathrm{a}$ and $4 \mathrm{~b}$ ). This type of cognitive strategy proved ineffective for regulating anxiety and affecting perception. Thus, 
existing literature does not specify an anxiety-reduction strategy that impacts visual perception consistently.

Furthermore, no research has statistically demonstrated how such regulation strategies influence perception. The current research tests whether providing participants with implementation intentions will alter perceptions of distance during performance. While reduced levels of anxiety are conjectured to be the process by which some of the emotion regulation strategies tested affect perception of the environment, no research has empirically or statistically tested whether reductions in anxiety serve as the mediating variable. As such, it is possible that third variables that have a documented effect on perception, like mood, were instead responsible for effects (Riener, Stefanucci, Proffitt, \& Clore, 2011). In the present research, we specifically test whether reduced levels of anxiety mediate any effect that implementation intentions have on perceptual distance estimates.

\section{Distance Perception and Distinction From a Model of Action-Specific Perception}

We predicted a relationship between perception and performance based on the fact that actual distance to goal-relevant targets in the environment can hinder performance. When targets are physically far away, like the hole to a golfer who is putting from the edge of the green or the hoop to a basketball player at half court, the task is more difficult and performance suffers. This common intuition has been supported empirically. Indeed, in one study, researchers manipulated task ease by changing the distance to a golf hole; although not the central finding of the research, participants who stood farther away from a golf hole were less successful at putting than those who stood closer to the hole (Witt, Linkenauger, Bakdash, \& Proffitt, 2008, Study 2). Likewise, dart performance suffered when dart throwers stood farther away from the dartboard compared with when they stood close (Edwards, Waterhouse, Atkinson, \& Reilly, 2007). Extrapolating from the fact that the actual proximity of goalrelevant targets influences performance on physical tasks, we predicted that perceived proximity to a goal-relevant target might similarly relate to performance. Specifically, when a target is perceived as closer and the task seems subjectively easier, individuals may perform better than when the target is perceived as farther away.

Prior research has tested one direction of this proposed relationship between perception and performance (Witt, 2011). Golfers who were more successful putters subsequently perceived the size of a golf hole as larger in comparison with golfers who performed worse (Witt et al., 2008). Similarly, successful football kickers perceived the size of field goal posts as larger than less successful kickers (Witt \& Dorsch, 2009). This line of research, referred to as the actionspecific account, suggests that performance has downstream consequences for perception. The self-selected nature of this type of research leaves open the question of whether perception can influence performance when perception is experimentally altered. For instance, some people are better at sports and may also happen to perceive elements of their world differently as a result. Thus, existing evidence supports one directional relationship between performance and perception and leaves unanswered several remaining questions.

The current research tests a different directional relationshipwhether and how perception affects performance. Previous research may appear to have suggested that perception does not predict performance (Wesp, Michelle, Gracia, \& Davis, 2004; Witt \& Dorsch, 2009). However, this conclusion was based on the lack of a direct effect of perception on performance when perception was measured prior to performance. We believe instead that perception will predict performance only when mediating mechanisms that indirectly link perception to performance are taken into account. In the present research, we test whether subjective ease acts as one mechanism that indirectly links effects of perception on performance. We expect that perceiving the environment in less extreme ways will increase the subjective feeling of task ease and as a result improve performance. Furthermore, this research proposes a novel hypothesis - that implementation intentions can serve as one anxiety-reducing strategy to induce perceived proximity and improve performance through subjective task ease.

\section{Overview of Studies}

Two studies tested whether participants who regulated their anxiety would perceive a goal-relevant target as closer and perform better on a physical task than participants who did not. In Study 1, participants putted a golf ball. The hole served as a goal-relevant target, and we measured performance by counting the number of putts that landed in the hole. Participants used implementation intentions to regulate anxiety or did not use a self-regulation strategy (control condition) before estimating the distance to the hole and putting. Study 2 conceptually replicated this design but varied the performance domain; participants threw darts. A dartboard served as the goal-relevant target, and we measured the number of darts thrown into the center of the dartboard. Prior to estimating the distance to a dartboard and throwing darts, participants formed implementation intentions to regulate anxiety, did not use a self-regulation strategy (control condition), or simply held the goal to regulate anxiety but were not given a specific strategy to do so.

In both studies, we predicted that implementation intentions would reduce participants' anxiety. Furthermore, we expected that participants who formed implementation intentions would perceive goal-relevant targets as closer and perform better on the tasks than participants who did not use this strategy. In both studies, to allay concerns that "postperceptual" processes, including cognitive judgment, guided distance 
estimates, we assessed perceptions of distance with standard perceptual measures used by vision researchers. Specifically, we avoided measures that required participants to translate perceptual experiences into numeric judgments, and instead used measures that required perceptual matching (Philbeck \& Loomis, 1997; Witt, Proffitt, \& Epstein, 2004).

Moreover, in Study 2, we tested the mechanism by which perception leads to improved performance. We proposed that perceived proximity leads to improved performance because participants construe the task as subjectively easier. Just as actual proximity predicted improves performance through actual or objective task ease (Edwards et al., 2007; Witt et al., 2008), we expected that perceived proximity would similarly improve performance through subjective task ease. It is important to note that we expected perception to influence performance indirectly through subjective task ease. In other words, we did not necessarily expect that perceived proximity to a goal-relevant target would alone have a direct effect on performance. Given that we did not predict a direct relationship between perception and performance, our model did not posit that people needed to be accurate in their perceptions of the environment to perform well. Instead, we predicted that they simply needed to perceive goal-relevant targets as closer to subjectively construe the task as easier, which in turn would affect task performance.

\section{Study I}

In Study 1, we tested whether regulating anxiety by forming implementation intentions would lead participants to perceive the goal-relevant target, a golf hole, as closer and perform better when putting. All participants had a goal to perform well at putting and were filmed throughout the experiment to induce anxiety. Participants were randomly assigned to either form implementation intentions or form no strategy to regulate their anxiety. As previous research tested a reverse directional relationship, whereby performance influences perception, we also included a timing manipulation. We manipulated whether participants estimated distance to the golf hole either before or after completing a putting task.

\section{Method}

Participants. Participants $(N=48)$ were New York University (NYU) undergraduates recruited through the department participant pool, as well as individuals from the community who were recruited via advertisements and email (37 women; $M_{\text {age. }}=23$ years, age range $=19-59$ years $)$. Participants received either research credit or US\$10 for their participation. All participants had normal or corrected-to-normal vision. The study used a 2 (Anxiety-Regulation Strategy: implementation intention, no strategy) $\times 2$ (Timing of Perceptual Estimate: before putting, after putting) betweensubjects design.
Procedure. In the lab, an experimenter informed participants that they would take part in a study on golfing techniques. To induce anxiety and record participants' behaviors, the experimenter turned on a video camera and emphasized that the camera would tape participants throughout the experiment. To further induce anxiety, the experimenter stated that the NYU golf team would later rate, critique, and closely study these films to improve its own putting skills. Participants self-reported their putting experience using a 7-point scale $(1=$ not at all experienced; $7=$ very experienced $)$.

Information about negative states. To ensure that all participants had the same insight into what they might experience during the putting task, the experimenter read two lists to participants. The experimenter first read aloud a list of anxiety-related negative states that participants might experience during the task, such as feeling nervous, distracted, and tense, which were drawn from sports psychology research (Anshel \& Anderson, 2002; Ryska, 1998) and research using implementation intentions to reduce negative states (Achtziger et al., 2008). To provide all participants with possible ways to regulate anxiety, the experimenter then read aloud a second list comprising common ways to regulate negative states, like taking deep breaths, focusing on the task at hand, and telling oneself to remain calm (Anshel \& Anderson, 2002). All participants, regardless of self-regulation condition, heard the information contained in both lists.

Although all participants heard the list of negative states and ways to regulate those states, only those participants randomly assigned to the implementation intention condition $(n=24)$ were explicitly instructed about how to form if-then plans. Participants randomly assigned to the no-strategy condition $(n=24)$ received no further instructions on how to regulate anxiety and served as the control group.

Strategy formation. Participants assigned to form implementation intentions created if-then plans to regulate the negative states they could experience during the putting task. Specifically, participants formed personally relevant implementation intentions; personalized implementation intentions have the greatest impact in helping to overcome states that block goal progress (Adriaanse et al., 2010). To create personalized implementation intentions, the experimenter told participants to select the four negative states that they anticipated would have the most adverse effect on their performance during the putting task. Next, the experimenter asked participants to pick one strategy to help regulate each negative state. To fully endorse and internalize the plans, participants wrote out in an if-then format each negative state with the strategy they had selected to help regulate it (e.g., "If I feel irritated, then I will tell myself to relax"), and repeated each plan in their head. Participants assigned to the no-strategy control condition continued to the next part of the study without receiving instructions on how to form a strategy to regulate anxiety.

Goal inducement. To ensure that all participants had a goal to perform well on the task, the experimenter induced an 


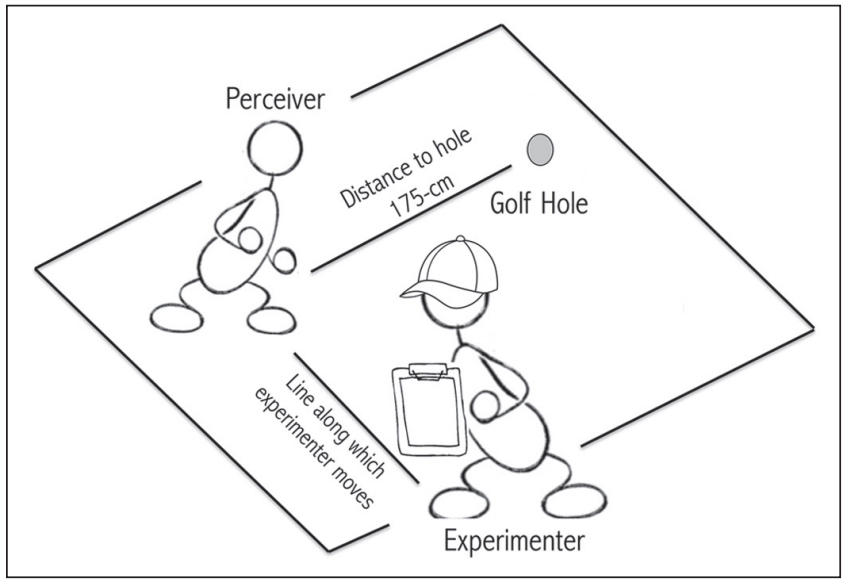

Figure I. Visual matching task used to measure perceived distance to the golf hole in Study I.

explicit putting goal by explaining that participants would have six attempts to putt a golf ball into a hole. If they successfully made three putts in the hole, they would receive candy and entry into a raffle for US $\$ 100$. To ensure that the anxiety-regulation strategy did not differentially affect the strength of participants' goals, participants completed a questionnaire before putting that asked them to rate their goal commitment, goal strength, and the predicted effort they would put into achieving their goal using 7-point scales (e.g., $1=$ not committed $; 7=$ very committed ).

Perceived distance and performance measures. Finally, we measured participants' perceptions of the distance to the hole and actual putting performance. We manipulated the timing of the distance estimate, such that participants were randomly assigned to estimate the distance to the hole either before $(n=24)$ or after $(n=24)$ putting six shots. To measure perceived distance to the golf hole, participants used a visual matching technique (Stefanucci \& Storbeck, 2009; Teachman et al., 2008). The putting mat was $175-\mathrm{cm}$ long, with the hole situated at the end of the putting mat opposite from the participant. All participants stood at the tee of the putting green. The golf mat and line of sight to the experimenter formed a $90^{\circ}$ angle with the participant serving as the vertex. As shown in Figure 1, to estimate perceived distance to the hole, participants matched the distance separating themselves from the experimenter with the distance separating themselves from the hole. To match these two distances, participants directed the experimenter to move closer or farther from themselves until the distance between themselves and the experimenter was equal to the distance between themselves and the hole. The participant could look to the hole throughout the visual matching task and continually reposition the experimenter until they were satisfied with their decision. The distance from the participant's chest to the experimenter's chest was then measured in centimeters with a tape measure. Participants who perceived the hole as close would have positioned the experimenter closer to themselves than participants who perceived the hole as far.

After completing the distance estimates and putting task, all participants were thanked and debriefed. No participants reported suspicion about the cover story concerning why they were being filmed.

Anxiety coding. To examine whether participants who formed implementation intentions were successful at regulating and reducing anxiety, two raters blind to hypotheses and condition coded the videotapes of participants for anxious behavior - a strategy for measuring anxiety that removes demand qualities in self-reported states that might artificially alter participants' reports of anxiety. Raters viewed silent videos starting 5 min before participants putted. Seven videos could not be coded due to camera malfunctions (camera malfunction did not vary with condition). ${ }^{1}$ A primary rater coded the full set of videos, and a secondary rater coded a subset $(22 \%)$. Each rater used a 1 (not at all) to 5 (a great deal) scale to indicate how anxious participants appeared in the videos. Each coder made an anxiety rating after every 1 min of footage. The correlation between raters' scores met acceptable standards, $r(45)=.76$. Because each participant had five ratings reflecting anxiety from each minute of taping, we created a composite score of anxiety for each participant. Within each minute, we averaged the two raters' anxiety scores. Next, we computed the mean across the five 1-min intervals $(\alpha=.83)$ to obtain an aggregate index of anxiety for each participant.

\section{Results}

Goal Strength and Experience. There was no effect of anxietyregulation strategy on self-reported goal strength, $t(46)=$ $-.69, p=.49$, level of goal commitment, $t(46)=.57, p=.58$, predicted effort put into achieving the goal, $t(46)=-.64, p=$ .53 , or putting experience, $t(46)=-.12, p=.91$. Thus, all participants, regardless of anxiety-regulation strategy, held equally strong performance goals.

Anxious Behavior. Adjusting for self-reported putting experience, participants who formed implementation intentions to regulate anxiety $(M=2.84, S D=.95)$ were less anxious than participants who did not have a strategy $(M=3.54, S D=$ $.68)$, as evidenced in the coded video recordings, $F(1,38)=$ $7.01, p=.01$. Thus, the formation of implementation intentions led participants to overtly express less anxious behavior prior to performing the putting task.

Perceived Distance. To test the prediction that participants who formed implementation intentions would view the hole as closer than participants who did not have a strategy, we conducted a 2 (Anxiety-Regulation Strategy: implementation intention, no strategy) $\times 2$ (Timing of Perceptual Estimate: before putting, after putting) ANCOVA predicting distance estimates. We included self-reported putting experience as a 
Table I. Mean (SD) Perceived Distance $(\mathrm{cm})$ to Hole and Performance as a Function of Regulation Condition and Timing of the Perceptual Estimate in Study I.

\begin{tabular}{lcr}
\hline & \multicolumn{2}{c}{ Regulation condition } \\
\cline { 2 - 3 } & $\begin{array}{c}\text { Implementation } \\
\text { intention }\end{array}$ & No strategy \\
\hline Distance estimate & & \\
$\quad$ Estimate prior to putting & $\mathrm{I} 58.17(\mathrm{I} .95)$ & $\mathrm{I} 9 \mathrm{I} .42(20.26)$ \\
Estimate after putting & $\mathrm{I} 1 \mathrm{I} .50(2 \mathrm{I} .99)$ & $\mathrm{I} 1 \mathrm{I} .42(27.33)$ \\
$\quad$ Total & $\mathrm{I} 59.83(19.7 \mathrm{I})$ & $\mathrm{I} 86.42(24.07)$ \\
Performance & & \\
$\quad$ Estimate prior to putting & $2.77(2.08)$ & $\mathrm{I} .92(\mathrm{I} .68)$ \\
Estimate after putting & $2.89(\mathrm{I} .19)$ & $\mathrm{I} .50(\mathrm{I} .62)$ \\
Total & $2.83(\mathrm{I} .66)$ & $\mathrm{I} .7 \mathrm{I}(\mathrm{I} .63)$ \\
\hline
\end{tabular}

Table 2. Partial Correlations Among Dependent Variable in Study I, Adjusting for Putting Experience.

\begin{tabular}{lcc}
\hline Measure & 1 & 2 \\
\hline I. Coded anxiety in video & - & \\
2. Perceived distance & $.37^{* *}$ & - \\
3. Performance & -.02 & -.12 \\
\hline
\end{tabular}

$* * p<.01$.

covariate. As expected, this analysis revealed a significant main effect of regulation strategy, $F(1,43)=17.52, p<.01$. As shown in Table 1, participants who formed implementation intentions $(M=159.83, S D=19.71)$ perceived the hole as closer than did participants assigned to the no-strategy control group $(M=186.42, S D=24.07)$. Neither the main effect of timing of the distance estimate, $F(1,43)=.25, p=.62$, nor the interaction effect between regulation strategy and timing, $F(1,43)=.75, p=.39$, were significant.

In addition, as shown in Table 2, participants who expressed less anxiety leading up to the putting task perceived the golf hole as closer. To check whether participants who formed implementation intentions perceived the golf hole as closer because they were experiencing less anxiety, we conducted a bootstrapped mediation analysis. We tested whether regulation strategy (effect coded such that $1=$ implementation intention and $-1=n o-$-strategy control) predicted perceived distance through anxiety. Self-reported putting experience was again included as a covariate. The $95 \%$ bias-corrected confidence interval (CI) of the indirect effect did not contain $0[-8.73,-.01]$, indicating that the mediation was significant (Preacher \& Hayes, 2004). These results suggest that forming implementation intentions, rather than having no strategy, led to perceived proximity through a reduction in anxious behaviors.

Performance. We also predicted that forming implementation intentions would lead to better putting performance in comparison with not forming a strategy. We conducted a 2 (Anxiety-Regulation Strategy: implementation intention, no strategy) $\times 2$ (Timing of Perceptual Estimate: before putting, after putting) ANCOVA predicting the number of shots successfully made in the hole. We again included putting experience as a covariate. As expected, the analysis yielded a significant main effect of regulation strategy, $F(1,43)=$ $5.45, p=.02$. As shown in Table 1, participants who had formed implementation intentions $(M=2.83, S D=1.66)$ putted into the hole more often than participants in the nostrategy control group $(M=1.71, S D=1.63)$. Neither the main effect of timing, $F(1,43)=.17, p=.68$, nor the interaction effect between emotion regulation strategy and timing were significant, $F(1,43)=.04, p=.84$.

In sum, participants who formed implementation intentions to regulate their anxiety displayed less anxious behavior, saw the hole as closer, and performed better on the putting task in comparison with the no-strategy control group. In addition, these effects controlled for putting experience and were not the result of differences in levels of goal commitment, goal strength, or predicted effort.

\section{Study 2}

Study 2 had four objectives. First, Study 2 added a control condition to equate the strength of goals to reduce anxiety. In Study 1, participants who formed implementation intentions may have held the goal to reduce anxiety more strongly than did participants in the no-strategy condition. In other words, the effects may have been driven not by the strategy itself, but by an increased desire to regulate anxiety in the implementation intention condition. Thus, we added an anxietyreduction condition in Study 2, akin to an emotion suppression condition (Gross, 2001), such that some participants held the goal to reduce anxiety but received no specific instructions on how to most effectively ward off anxiety. Participants who held the goal to reduce anxiety and those who used implementation intentions should hold equally strong anxietyreduction goals. However, because participants who only held the goal to reduce anxiety must consciously and effortfully consider how to reduce anxiety (Achtziger et al., 2008; Gross, 1998, 2001), we expected the goal would not reduce anxiety as well as implementation intentions.

Second, we used a different technique to assess perceptions of distance. Because the matching task in Study 1 required participants to move the experimenter, it is possible that the anxiety individuals felt produced the desire for increased distance between participants and the experimenter (Brady \& Walker, 1978). In Study 2, we controlled for the possibility that the desire to create interpersonal distance affected the dependent variable by eliminating the involvement of the experimenter. Specifically, participants completed a visual matching task in which they moved themselves toward or away from the target until the distance between themselves and the target equaled the distance between two 
given markers (Balcetis \& Dunning, 2010; modified from Philbeck \& Loomis, 1997; Witt et al., 2004).

Third, Study 2 controlled for the possibility that time delays reduced anxiety. In Study 1, participants in the implementation intention condition, but not the control condition, experienced a time delay of several minutes between hearing about negative states they might experience and performing the task, which may have reduced anxiety in and of itself (Zillman \& Cantor, 1976). In Study 2, all participants experienced a time delay of several minutes.

Fourth, in Study 2 we tested one process by which perceptions of proximity might improve performance. Specifically, we tested whether perceived proximity improves performance by increasing subjective feelings of task ease. Actual proximity to a goal-relevant target should lead participants to construe a physical task, such as putting or throwing darts, as easier and enhance performance on the task. ${ }^{2}$ As such, we conjectured that simply the perceptual experience of proximity might similarly lead a performer to construe a physical task as subjectively easier. In addition, given the relationship between subjective feelings of task ease and better sports performance (Rejeski \& Lowe, 1980), construing the task as subjectively easier might lead to improved task performance. Thus, we predicted that perceived distance might indirectly improve performance through increases in subjective task ease.

\section{Method}

Participants. Ninety-three NYU undergraduates (61 women; $M_{\text {age }}=19.8$ years, age range $=18-29$ years) participated in exchange for either research credit or US\$10. Data from three additional participants were excluded from analyses because one participant did not have normal or corrected-tonormal vision, and two participants' distance estimates were larger than three $S D$ s above the mean. Overall, the study used a 3 (Anxiety-Regulation Strategy: implementation intention, anxiety-regulation goal only, no strategy) $\times 2$ (Visual Matching Start Location: near target, far from target) between-subjects design.

\section{Procedure}

Information about negative states and strategy formation. Participants learned that they would complete a study on hand-eye coordination measured through dart throwing. To increase anxiety and task investment, we filmed participants during their performance and indicated that these tapes would be rated by a graduate student who specializes in intelligence testing, a physiology psychologist who specializes in motor movement, and a member of the New York Dart Organization. Participants indicated their experience in dart throwing using a 7 -point scale $(1=$ not at all experienced $;=$ very experienced .

As in Study 1, all participants received information describing the negative states they might experience and the strategies people might use to manage these states. Participants randomly assigned to the implementation intention condition $(n=31)$ received instructions regarding how to form if-then plans as in Study 1 and completed the formation process with the experimenter. Participants randomly assigned to the anxiety-regulation goal condition $(n=30)$ formed, wrote down, and repeated aloud from memory the strategy "I will try my hardest to not let any feelings bother me so I can perform the dart throwing task well!" (adapted from Achtziger et al., 2008). Participants randomly assigned to the no-strategy condition $(n=32)$ continued to the next part of the study without receiving any further instructions on how to regulate anxiety.

Anxiety coding. To measure participants' anxiety prior to throwing darts, participants completed a thought-listing task. Participants used 2 min to prepare themselves for the dartthrowing task. During this time, they said aloud everything they were feeling or thinking relevant to preparing for the task. Their thoughts were audio-recorded. Participants could report their experience of negative feelings or anxiety without explicitly being asked to do so, thereby reducing demand effects. Two raters blind to hypotheses and condition coded the audio recordings for anxiety. Fifteen percent $(n=14)$ of the recordings could not be coded due to recording complications or the participant's decision not to speak during the time recorded (recordings that could not be coded did not vary with condition). ${ }^{3}$ A primary rater coded the full set of recordings, and a secondary rater coded a subset (42\%). Each rater used a 1 (not at all) to 5 (a great deal) scale to code the extent to which participants sounded uncomfortable, nervous, tense, and anxious. The correlations between raters' scores for each variable ranged from $r(33)=.72$ to .76. From the scores, we created a composite measure of anxiety for each participant. To create this score, we first averaged together the two raters' scores within each variable (e.g., averaging the raters' scores for how uncomfortable a participant sounded). Next, we averaged scores across the four anxiety variables for each participant $(\alpha=.78)$.

Goal inducement. All participants received a performance goal. They learned that previous participants hit the center target of the dartboard, on average, 4 out of 6 times. In addition, to guarantee that all participants had a strong goal to perform well, we provided participants with information regarding how the dart-throwing task was serving as a new intelligence test the lab was ostensibly developing (McClelland, 1973). Participants read a short history of standardized intelligence tests emphasizing that intelligence tests based on hand-eye coordination were an important measure of achievement and aptitude. Participants read that if they performed well at the task, that would indicate they had good hand-eye coordination and therefore high intelligence.

To ensure that the anxiety-regulation strategy did not affect qualities of the performance goal, participants reported their performance goal commitment and goal strength, and predicted the effort they would put into the task using 7-point 


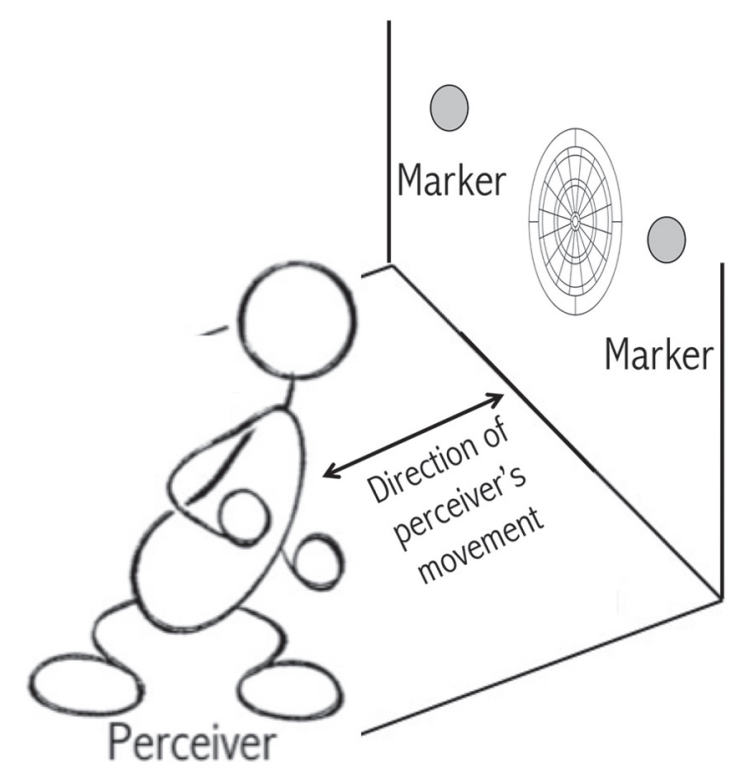

Figure 2. Visual matching task used to measure perceived distance to the dartboard in Study 2.

scales (e.g., 1 = not committed; 7 = very committed). To measure subjective task ease, participants reported the anticipated ease at reaching the goal of hitting four darts in the center of the dartboard using a 7-point scale $(1=$ not at all easy; 7 = very easy).

Perceived distance and performance measures. To measure perceptions of distance to the dartboard, participants completed a visual matching task that has been used in past research (Balcetis \& Dunning, 2010; Witt et al., 2004). Two markers were affixed to a wall $180-\mathrm{cm}$ apart. One marker was on each side of the dartboard. As shown in Figure 2, participants faced the dartboard. The experimenter told participants to look at the two markers on the wall and gain a sense of how far apart they thought the two markers were. The experimenter then asked participants to match the distance between themselves and the dartboard with the distance between the two markers on the wall. Participants could move forward and backward until they believed they equated the distance between themselves and the dartboard to the distance between the two pieces of tape on the wall. The experimenter then measured the distance from the participant's chest to the dartboard with a tape measure. To counterbalance starting position and the need to move forward or backward, participants were randomly assigned to begin their distance estimate standing either near $(60 \mathrm{~cm}$; $n=47$ ) or far (285 cm; $n=46$ ) from the dartboard. Importantly, because participants matched their perceived distance from the dartboard to the distance between the two markers, a participant who perceived the dartboard as closer would actually need to stand farther away from the dartboard than a participant who perceived the dartboard as farther away. Thus, higher distance scores actually indicated that the participant perceived greater proximity between themselves and the dartboard.

Finally, to measure performance, all participants threw six darts while standing $260-\mathrm{cm}$ away from the dartboard, and were then thanked and debriefed. No participants reported suspicion about the cover story concerning why they were being filmed.

\section{Results}

Goal Strength and Experience. Participants in all three anxietyregulation conditions did not differ in terms of performance goal strength, $F(2,90)=.47, p=.63$; level of goal commitment, $F(2,90)=.62, p=.54$; predicted effort put into achieving the goal, $F(2,90)=.29, p=.75$; or dart-throwing experience, $F(2,90)=.91, p=.41$. Regardless of anxietyregulation condition, participants held equally strong performance goals.

Anxious Language. To test whether forming implementation intentions reduced participants' anxiety prior to throwing the darts, we analyzed the anxiety ratings of the audio recordings. We predicted that the anxiety-regulation goal and nostrategy groups would not significantly differ on the anxiety composite. We used contrast weights of -2 for the implementation intention group and +1 for the anxiety-regulation goal and no-strategy groups. Adjusting for dart-throwing experience, a planned contrast comparing the anxiety scores of the implementation intention group to those of the anxiety-regulation goal and no-strategy group was significant, $F(1,76)=6.96, p=.01$. Confirming our predictions, participants who formed implementation intentions sounded significantly less anxious than participants who held an anxiety-regulation goal or no strategy (see Table 3 ).

Perceived Distance. We predicted that participants who formed implementation intentions would perceive the dartboard as closer than participants who held an anxiety-regulation goal only or who had no anxiety-regulation strategy. We conducted a 3 (Anxiety-Regulation Strategy: implementation intention, anxiety-regulation goal, no strategy) $\times 2$ (Visual Matching Start Location: near, far) ANCOVA predicting distance estimates. Self-reported dart-throwing experience was included as a covariate. Higher numbers indicate perceiving the dartboard as closer (i.e., greater perceived proximity). The analysis revealed the expected main effect of strategy type, $F(2,86)=5.13, p<.01$. There was no effect of starting location, $F(1,86)=2.41, p=.12$. The interaction between strategy type and estimate start point was not significant, $F(2,86)=.62, p=.54$.

Because we predicted that the distance estimates would be the smallest within the implementation intention condition and would not differ between the anxiety-regulation 
Table 3. Mean (SD) Values of Anxiety, Performance, Ease, and Distance as a Function of Regulation Condition in Study 2.

\begin{tabular}{lccc}
\hline & \multicolumn{3}{c}{ Regulation condition } \\
\cline { 2 - 4 } & $\begin{array}{c}\text { Implementation } \\
\text { intention }\end{array}$ & $\begin{array}{c}\text { Anxiety-regulation } \\
\text { goal }\end{array}$ & $\begin{array}{c}\text { No-strategy } \\
\text { control }\end{array}$ \\
\hline Anxiety & $2.21_{\mathrm{a}}(0.82)$ & $2.66_{\mathrm{b}}(0.68)$ & $2.59_{\mathrm{b}}(0.63)$ \\
Performance & $2.8 \mathrm{I}_{\mathrm{a}}(1.58)$ & $1.90_{\mathrm{b}}(1.37)$ & $1.94_{\mathrm{b}}(1.24)$ \\
Ease & $3.32_{\mathrm{a}}(1.22)$ & $2.83_{\mathrm{b}}(1.09)$ & $2.56_{\mathrm{b}}(0.98)$ \\
Perceived proximity & & & \\
$\quad$ Estimate started close & $227.48(45.64)$ & $186.81(33.81)$ & $198.48(28.64)$ \\
Estimate started far & $229.63(28.14)$ & $198.50(61.02)$ & $223.99(35.58)$ \\
Total & $228.52_{\mathrm{a}}(37.58)$ & $192.65_{\mathrm{b}}(48.83)$ & $211.24_{\mathrm{b}}(34.32)$ \\
\hline
\end{tabular}

Note: Greater values on perceived proximity indicate participants stood further from the dartboard, meaning they perceived the dartboard as closer. Within rows, values with different subscripts significantly differ at $p<.05$ in the planned contrasts.

Table 4. Partial Correlations Among Dependent Variable in Study 2, Adjusting for Dart-Throwing Experience.

\begin{tabular}{llll}
\hline Measure & 1 & 2 & 3 \\
\hline I. Coded anxiety in voice & - & & \\
2. Perceived proximity & $-.24 *$ & - & \\
3. Performance & -.14 & .06 & - \\
4. Ease & $-.39 * * *$ & $.22^{*}$ & $.29 * *$ \\
\hline
\end{tabular}

$*_{p}<.05 . * * p<.01 . * * * p<.001$.

goal and no-strategy groups, we conducted a planned contrast (weights: implementation intention $=-2$, anxiety-regulation goal $=+1$, no strategy $=+1$ ) that included dart-throwing experience as a covariate. This contrast was significant, $F(1$, $90)=7.04, p=.009$. As shown in Table 3, participants who formed implementation intentions perceived the dartboard as significantly closer than both participants who held an anxietyregulation goal and those who had no strategy to reduce anxiety. In addition, as shown in Table 4, participants who expressed less anxiety in their voice recordings perceived greater proximity to the dartboard.

To test whether participants who formed implementation intentions perceived greater proximity to the dartboard because they experienced less anxiety, we conducted a bootstrapped mediation analysis. We tested whether regulation strategy predicted perceived proximity through anxiety. Self-reported dart-throwing experience was included as a covariate. The $95 \%$ bias-corrected CI of the indirect effect did not contain $0[-7.03,-.01]$, indicating that the mediation was significant. These results suggest that regulating anxiety by forming if-then plans led participants to perceive a goalrelevant target (the dartboard) as closer than did forming an anxiety-regulation goal or using no strategy.

Performance. We also predicted that participants who formed implementation intentions would perform better on the task than the anxiety-regulation goal and no-strategy groups. We conducted a planned contrast testing the implementation

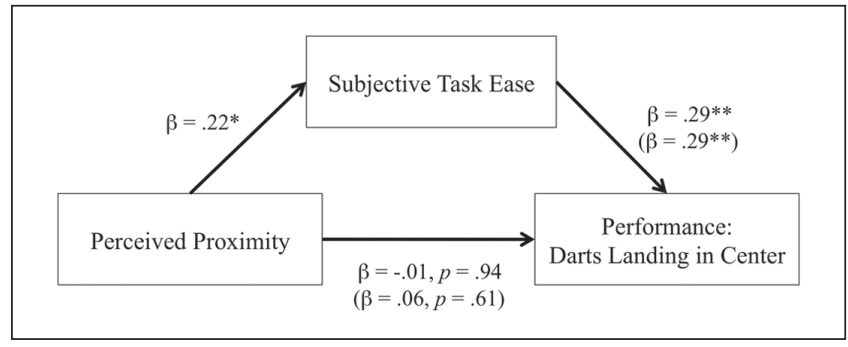

Figure 3. Mediation model in Study 2.

Note: Perceived proximity indirectly improved performance through increased subjective task ease. Values in parentheses represent direct relationships; values without parentheses represent relationships after including all variables in the model.

$*_{p}<.05 . *_{p}<.01$

intention group against the anxiety-regulation goal and nostrategy group, using the same weights as above, with dart-throwing performance as the dependent variable. Dartthrowing experience was included as a covariate. The contrast was significant, $F(1,90)=8.25, p=.005$. Confirming our predictions, participants who formed implementation intentions threw more darts that landed in the center circle than both participants who held an anxiety-regulation goal and who had no strategy (see Table 3 ). These results replicate and extend the findings of Study 1; forming implementation intentions to regulate anxiety can enhance performance on a physical task relative to having simply an anxiety-regulation goal or no strategy.

Ease of Goal Achievement. We also predicted that participants who formed implementation intentions would judge the task as subjectively easier than the other two groups. Adjusting for dart-throwing experience, a planned contrast testing the implementation intention group (contrast weight $=-2$ ) against the anxiety-regulation goal and no-strategy group (each contrast weight $=+1$ ) with subjective ease of reaching the dart-throwing goal as the dependent variable was significant, $F(1,90)=8.43, p=.005$. As shown in Table 3, participants who formed implementation intentions evaluated the task as easier than did participants who had a regulation goal or no strategy. These results indicate that participants who formed if-then plans construed the task as easiest to complete.

Mediation Analysis. Finally, we tested whether perceived proximity improved performance because it made the task seem easier. As shown in Figure 3, participants who perceived the dartboard as closer also believed that it would be easier to reach their goal. In addition, participants who perceived reaching their goal to be easier landed more darts in the center circle of the dartboard. Although we did not find a direct relationship between perceived distance to the dartboard and performance on the dart-throwing task, this direct effect is not a prerequisite for testing mediation with bootstrapping procedures (Preacher 
\& Hayes, 2004; Shrout \& Bolger, 2002). Thus, as shown in Figure 3, we tested the effect of the indirect pathway from perceived proximity of the dartboard to dart-throwing performance as mediated by subjective task ease. Dart-throwing experience was included as a covariate. The bootstrapping procedure yielded a $95 \%$ CI that did not contain 0 [.0002, .0058], indicating that the mediation was significant. Perceiving the target as closer enhanced performance indirectly through increasing the subjective ease of the task. ${ }^{4}$

In sum, our results are consistent with the conclusions that participants who created implementation intentions, and thus effectively regulated their anxiety, perceived the dartboard as closer and in turn performed better because they construed the task as easier. In addition, these effects were not due to simply holding any type of anxiety-regulation goal, but rather were specific to forming an implementation intention strategy.

\section{Discussion}

Two studies suggest that during a physical task, implementation intention strategies can effectively reduce anxiety, which in turn leads to perceived proximity to a goal-relevant target and better performance. Participants who formed implementation intentions to regulate anxiety perceived a golf hole (Study 1) and dartboard (Study 2) as closer and were more successful performers than both participants who had no strategy (Studies 1-2) and participants who simply held the goal to regulate their anxiety but did not use an effective strategy to do so (Study 2). Furthermore, a mediation analysis suggested that perceiving the dartboard as closer led participants to perform better on the task because it made the task seem easier. Using various distance perception measures, we documented the effects of and processes behind the influence of a specific anxiety-reduction strategy on perception and performance.

\section{Perceived Proximity and Subjective Task Ease}

The present research adds to a developing theory of emotion, embodied perception, and action (Stefanucci \& Proffitt, 2009; Stefanucci \& Storbeck, 2009). In two studies, reducing anxiety led participants to see a goal-relevant target as closer, to construe the task as easier, and to perform better. The relationship between perception and performance was an indirect one, mediated through feelings of task ease. Future research should explore the underlying reasons why perceiving a task as easier leads to better performance. We conjecture that perceived proximity may make tasks feel subjectively easier because performers feel more confident about how to calibrate their motor movements (Klaus \& Norbert, 1978). Feelings of task ease may also reduce anxiety and thus free cognitive resources so that perceivers are able to more easily plan and enact their performance objectives. Subjective judgments of task ease may improve performance by way of multiple psychological and physical processes that as of yet are unexplored.

\section{Action-Specific Perception and the Reciprocal Relationship of Perception and Performance}

Some researchers have suggested a connection between action and perception. However, our model differs from past research in several ways. Existing research proposes specifically that performance changes perception of the environment (Witt, 2011). Better dart throwers subsequently see the dartboard as larger than worse throwers (Wesp et al., 2004), and successful batters see the baseball as larger than less successful batters (Witt \& Proffitt, 2005). In addition, past research that assessed perception before and after performance found that performance predicted perception but perception did not directly alter performance, when perception was measured first (Wesp et al., 2004; Witt \& Dorsch, 2009). Thus, past work exploring the relationship between perception and performance has focused almost entirely on a directional relationship whereby performance influences perception.

In this novel test, we found evidence of the reverse directional relationship, that perception can influence performance. After providing participants with strategies to reduce their anxiety, we found an indirect effect of perception on performance; perceived proximity improved performance by way of increased subjective task ease. Recently, Witt, Linkenauger, and Proffitt (2012) similarly conjectured, but did not test, the idea that changes in psychological feelings of confidence might mediate the action-perception link. Indeed, there may be psychological processes that mediate the links between perception and action.

In light of our findings and those of past work on actionspecific perception, one might ask if a consensus can be reached on whether perception influences performance or performance influences perception. Relationships demonstrated in past work and the current research suggest both possibilities (see also discussion in Witt et al., 2012) of which future research should test nuances. We conjecture that it is likely that directional effects may occur through separate mechanisms and across different time frames. For instance, the current studies demonstrated that perception indirectly influenced performance. Perceived proximity following an anxiety-regulation strategy led participants to feel that the task was easier and helped them perform better. It is possible that construing the task as easier freed up resources to focus on the task, allowed participants to think through their actions without distracting thoughts about task difficulty, and enabled participants to feel more confident in their actions (Woodman \& Hardy, 2003). Thus, perception may influence performance specifically by way of increased cognitive resources and feelings of confidence.

Alternatively, the relationship from performance to perception (Witt, 2011; Witt \& Dorsch, 2009) may occur 
through other processes. Performance may exert an influence on perception through learning mechanisms that must unfold over time. For instance, past research has shown that people who perform well at field goal kicking subsequently perceived the field goal poles as further apart (Witt \& Dorsch, 2009). An excellent football kicker might consistently kick the ball toward the dead center, between the field goal poles. Little variance in the placement of the kick relative to the poles might lead to the illusion that the poles are very far apart. In this case, performance may influence perception because participants learn over time, through their experiences, that the poles must be quite far apart. Future research aimed at developing comprehensive models of how perception and performance reciprocally influence each other might need to consider multiple mechanisms and time frames.

\section{Regulation Strategies}

In the present research, participants formed implementation intentions to regulate their anxiety. Although other strategies, like cognitive reappraisal, may help prevent the experience of anxiety, implementation intentions may serve as a uniquely effective form of anxiety reduction or prevention. Implementation intentions are automatically deployed and less likely to deplete cognitive resources (Schweiger Gallo et al., 2009). As a result, implementation intentions, and other strategies that automatically control anxiety like preemptive mental simulation (Taylor, Pham, Rivkin, \& Armor, 1998), may be more successful at mitigating anxiety and improving performance than techniques that require conscious effort.

\section{On Accuracy and Performance}

One may wonder whether implementation intentions lead to accuracy in perception, and whether accuracy in perceiving distance in our studies improves performance. We are cautious in rendering conclusions regarding perceptual accuracy. First, our model does not predict a direct effect of perception on performance. Indeed, we find that perception leads to improved performance because perceived proximity increases subjective task ease. Thus, to the extent that perceived proximity makes a task feel easier, performance should be better; this does not necessarily require that perception is accurate.

Second, we are cautious when conjecturing about the accuracy of distance estimates, given that we are most likely testing effects that occur by way of a visual processing system not intended to produce accurate perceptual experiences. That is, the techniques we used assess perceptual processing that occurs by way of the explicit perceptual awareness system rather than the visuomotor system (see Witt \& Proffitt, 2007). The explicit awareness system promotes the planning of behaviors as people survey and consider how they will navigate the environment (Proffitt, Bhalla, Gossweiler, \&
Midgett, 1995). Juxtaposed against this, the visuomotor system calibrates the body to guide immediate muscle movements to promote optimal motor navigation (Bhalla \& Proffitt, 1999). The techniques we used to assess perception are those that assess explicit perceptual awareness rather than visuomotor processes that affect muscle movements. In addition, our measures of perception were generally collected prior to action, and thus are measures reflective of perceptual planning. Future research could expand the repertoire of measures used to assess distance perception to explore the effects within these two visual processing systems.

\section{Moderating Factors}

While we propose that reduced anxiety improves performance through processes implicating visual perception, in some instances, lower anxiety may not benefit performance. In fact, Yerkes-Dodson Law articulates that the relationship between anxiety and performance is not linear. There are optimal levels of anxiety or arousal, and not necessarily simply lower levels, at which athletes perform their best (Klavora, 1977).

In addition, some tasks may actually require high levels of anxiety or arousal for premium performance. For instance, performance on tasks that require focused attention and vigilance to cues of threat may improve as anxiety increases (e.g., Eysenck, 1992). Socially anxious individuals maintain the goal to divert attention from faces expressing emotions. As such, they are vigilant for these faces (Mansell, Clark, Ehlers, \& Chen, 1999). Furthermore, people who experience anxious arousal when viewing neutral Black faces presented quickly (30 ms) are highly attentive to those faces (Richeson \& Trawalter, 2008). Thus, increased anxiety in these instances actually directs attention toward goal-relevant targets and subsequently improves goal-relevant performance. Future research might test moderators of the effect of anxiety on goal-relevant performance and include such factors as the optimal level of arousal, approach or avoidance orientation of the goal, and the goal-relevance of the contents of the environment.

\section{Concluding Remarks}

We have shown that regulating anxiety in a performance scenario causes perceivers to see a goal-relevant target as closer, leading them to construe a task as easier, and ultimately enhancing their performance on that task. The present research brings to light the functional role perception plays during performance, how the subjective ease of the task can influence performance, and the possibility of a reciprocal relationship between perception and performance. In sum, our findings provide insight for future research exploring how regulation strategies may be used to induce shifts in perception and how perception can improve physical task performance. 


\section{Acknowledgments}

The authors would like to thank Peter Linder, Stephanie Vivar, Makenzie Lowery, and Tara Mathai-Davis for assistance in data collection, as well as Michael Poggioli, Kristin Schenider, Matt Riccio, Hannah Pakray, Nicholas Sosa, and Jenna Koroly for coding.

\section{Declaration of Conflicting Interests}

The author(s) declared no potential conflicts of interest with respect to the research, authorship, and/or publication of this article.

\section{Funding}

The author(s) received no financial support for the research, authorship, and/or publication of this article.

\section{Notes}

1. Seven videos could not be coded (four in the no-strategy control, and three in the implementation intention condition). There were not significant differences in the percentage of uncodable videos across conditions, $\chi^{2}(1)=.17, p=.68$.

2. Pilot data we collected suggested that a task is objectively easier and seems easier when the goal-relevant target is actually closer. In the pilot study, participants $(N=28)$ were motivated to throw darts to the center of a dartboard, and either stood close to (4-ft away) or stood farther away from (12 ft) the dartboard. Participants who stood closer landed more darts in the center ring $(M=5.3, S D=0.8)$, than did those who stood farther away $(M=1.5, S D=1.6), t(26)=7.92, p<.01$. In addition, those who stood closer reported that it felt easier to reach their goal $(M=$ $4.1, S D=1.8)$, than did those who stood farther away $(M=2.4$, $S D=1.1), t(26)=2.97, p<.01$.

3. Fourteen recordings could not be coded (4 in the no-strategy control, 6 in the regulation goal, and 4 from the implementation intention condition). There were no significant differences in the percentage of uncodable recordings across conditions, $\chi^{2}(2)=$ $.85, p=.65$.

4. Because both subjective task ease and perceived proximity to the dartboard were measured variables, we tested an alternative mediation model. We conducted a bootstrapped mediation analysis in which subjective task ease predicted performance on the dart-throwing task through perceived proximity to the dartboard. Dart-throwing experience was included as a covariate. The $95 \%$ bias-corrected confidence interval (CI) of the indirect effect contained $0[-.06, .06]$, indicating that this alternative mediation was not significant.

\section{References}

Achtziger, A., Gollwitzer, P. M., \& Sheeran, P. (2008). Implementation intentions and shielding goal striving from unwanted thoughts and feelings. Personality and Social Psychology Bulletin, 34, 381-393.

Adriaanse, M. A., Oettingen, G., Gollwitzer, P. M., Hennes, E. P., De Ridder, D. T. D., \& De Wit, J. B. F. (2010). When planning is not enough: Fighting unhealthy snacking habits by mental contrasting with implementation intentions (MCII). European Journal of Social Psychology, 40, 1277-1293.

Anshel, M. H., \& Anderson, D. I. (2002). Coping with acute stress in sports: Linking athletes' coping style, coping strategies, affect, and motor performance. Anxiety, Stress \& Coping: An International Journal, 15, 193-209.

Balcetis, E., \& Dunning, D. (2010). Wishful seeing: Desired objects are seen as closer. Psychological Science, 21, 147-152.

Baumeister, R. F., \& Showers, C. J. (1986). A review of paradoxical performance effects: Choking under pressure in sports and mental tests. European Journal of Social Psychology, 16, 361383.

Bayer, U. C., \& Gollwitzer, P. M. (2007). Boosting scholastic test scores by willpower: The role of implementation intentions. Self and Identity, 6, 1-19.

Beilock, S. L., \& Carr, T. H. (2001). On the fragility of skilled performance: What governs choking under pressure? Journal of Experimental Psychology: General, 130, 701-725.

Beilock, S. L., \& Gray, R. (2007). Why do athletes choke under pressure? In G. Tenenbaum \& R. C. Eklund (Eds.), Handbook of sport psychology (3rd ed., pp. 425-444). Hoboken, NJ: John Wiley.

Bhalla, M., \& Proffitt, D. R. (1999). Visual-motor recalibration in geographical slant perception. Journal of Experimental Psychology: Human Perception and Performance, 25, 1076-1096.

Brady, A., \& Walker, M. (1978). Interpersonal distance as a function of situationally induced anxiety. British Journal of Social Psychology, 17, 127-133.

Edwards, B., Waterhouse, J., Atkinson, G., \& Reilly, T. (2007). Effects of time of day and distance upon accuracy and consistency of throwing darts. Journal of Sports Science, 13, 1531-1536.

Eysenck, M. W. (1992). Anxiety: The cognitive perspective. Hillsdale, NJ: Lawrence Erlbaum.

Gollwitzer, P. M. (1993). Goal achievement: The role of intentions. European Review of Social Psychology, 4, 141-185.

Gollwitzer, P. M. (1999). Implementation intentions: Strong effects of simple plans. American Psychologist, 54, 493-503.

Gollwitzer, P. M., \& Sheeran, P. (2006). Implementation intentions and goal achievement: A meta-analysis of effects and processes. Advances in Experimental Social Psychology, 38, 69-119.

Gross, J. J. (1998). The emerging field of emotion regulation: An integrative review. Review of General Psychology, 2, 271-299.

Gross, J. J. (2001). Emotion regulation in adulthood: Timing is everything. Current Directions in Psychological Science, 10, 214-219.

Gross, J. J., \& Levenson, R. W. (1997). Hiding feelings: The acute effects of inhibiting positive and negative emotions. Journal of Abnormal Psychology, 106, 95-103.

Janelle, C. M. (2002). Anxiety, arousal, and visual attention: A mechanistic account of performance variability. Journal of Sports Sciences, 20, 237-250.

Klaus, S., \& Norbert, P. (1978). The influence of experience with a task on task preference, subjective uncertainty and causal explanations of success. Psychologische Beitrage, 20, 228-250.

Klavora, P. (1977). An attempt to derive inverted-U curves based on the relationship between anxiety and athletic performance. 
In D. M. Landers \& R. W. Christina (Eds.), Psychology of motor behavior and sport-1977 (pp. 369-377). Champaign, IL: Human Kinetics.

Lewis, B., \& Linder, D. (1997). Thinking about choking? Attention processes and paradoxical performance. Personality and Social Psychology Bulletin, 23, 937-944.

Mansell, W., Clark, D. M., Ehlers, A., \& Chen, Y. (1999). Social anxiety and attention away from emotional faces. Cognition \& Emotion, 13, 673-690.

Martens, R., Burton, D., Vealy, R. S., Bump, L. A., \& Smith, D. E. (1990). Development and validation of the Competitive State Anxiety Inventory-2. In R. Martens, R. S. Vealy, \& D. Burton (Eds.), Competitive anxiety in sport (pp. 117-190). Champaign, IL: Human Kinetics.

McClelland, D. C. (1973). Testing for competence rather than for “intelligence." American Psychologist, 28, 1-14.

Philbeck, J. W., \& Loomis, J. M. (1997). Comparison of two indicators of perceived egocentric distance under full-cue and reduced-cue conditions. Journal of Experimental Psychology: Human Perception and Performance, 23, 72-85.

Preacher, K. J., \& Hayes, A. F. (2004). SPSS and SAS procedures for estimating indirect effects in simple mediation models. Behavior Research Methods, Instruments, \& Computers, 36, 717-731.

Proffitt, D. R., Bhalla, M., Gossweiler, R., \& Midgett, J. (1995). Perceiving geographical slant. Psychonomic Bulletin \& Review, 2, 409-428.

Rachman, S., \& Cuk, M. (1992). Fearful distortions. Behavior Research and Therapy, 30, 583-589.

Rejeski, W. J., \& Lowe, C. A. (1980). The role of ability and effort in attributions of sports achievement. Journal of Personality, $48,233-244$.

Richards, J. M., \& Gross, J. J. (1999). Composure at any cost? The cognitive consequences of emotion suppression. Personality and Social Psychology Bulletin, 25, 1033-1044.

Richeson, J. A., \& Trawalter, S. (2008). The threat of appearing prejudiced and race-based attentional biases. Psychological Science, 19, 98-102.

Riener, C. R., Stefanucci, J. K., Proffitt, D. R., \& Clore, G. (2011). An effect of mood on the perception of geographical slant. Cognition \& Emotion, 25, 174-182.

Ryska, T. A. (1998). Cognitive-behavioral strategies and precompetitive anxiety among recreational athletes. Psychological Record, 48, 697-708.

Schweiger Gallo, I., Keil, A., McCulloch, K. C., Rockstroh, B., \& Gollwitzer, P. M. (2009). Strategic automation of emotion regulation. Journal of Personality and Social Psychology, 96, 11-31.

Shrout, P. E., \& Bolger, N. (2002). Mediation in experimental and nonexperimental studies: New procedures and recommendations. Psychological Methods, 7, 422-445.
Stefanucci, J. K., \& Proffitt, D. R. (2009). The roles of altitude and fear in the perception of heights. Journal of Experimental Psychology: Human Perception and Performance, 35, 424-438.

Stefanucci, J. K., Proffitt, D. R., Clore, G. L., \& Parekh, N. (2008). Skating down a steeper slope: Fear influences the perception of geographical slant. Perception, 37, 321-323.

Stefanucci, J. K., \& Storbeck, J. (2009). Don't look down: Emotional arousal elevates height perception. Journal of Experimental Psychology: General, 138, 131-145.

Taylor, S. E., Pham, L. B., Rivkin, I. D., \& Armor, D. A. (1998). Harnessing the imagination: Mental stimulation, self-regulation, and coping. American Psychologist, 53, 429-439.

Teachman, B. A., Stefanucci, J. K., Clerkin, E. M., Cody, M. W., \& Proffitt, D. R. (2008). A new mode of fear expression: Perceptual bias in height fear. Emotion, 8, 296-301.

Wesp, R., Michelle, P., Gracia, E. B., \& Davis, K. (2004). Observing and engaging in purposeful actions with objects influences estimates of their size. Perception \& Psychophysics, 66, 1261-1267.

Witt, J. K. (2011). Action's effect on perception. Current Directions in Psychological Science, 20, 201-206.

Witt, J. K., \& Dorsch, T. (2009). Kicking to bigger uprights: Field goal kicking performance influences perceived size. Perception, 38, 1328-1340.

Witt, J. K., Linkenauger, S. A., Bakdash, J. Z., \& Proffitt, D. R. (2008). Putting to a bigger hole: Golf performance relates to perceived size. Psychonomic Bulletin \& Review, 15, 581-585.

Witt, J. K., Linkenauger, S. A., \& Proffitt, D. R. (2012). Get me out of this slump! Visual illusions improve sports performance. Psychological Science, 23, 397-399.

Witt, J. K., \& Proffitt, D. R. (2005). See the ball, hit the ball: Apparent ball size is correlated with batting average. Psychological Science, 16, 937-938.

Witt, J. K., \& Proffitt, D. R. (2007). Perceived slant: A dissociation between perception and action. Perception, 32, 249-257.

Witt, J. K., Proffitt, D. R., \& Epstein, W. (2004). Perceiving distance: A role of effort and intent. Perception, 33, 577-590.

Woodman, T., \& Hardy, L. (2001). Stress and anxiety. In R. N. Singer, H. A. Hausenblaus, \& C. M. Janelle (Eds.), Handbook of sport psychology (2nd ed., pp. 290-318). New York, NY: John Wiley.

Woodman, T., \& Hardy, L. (2003). The relative impact of cognitive anxiety and self-confidence upon sports performance: A metaanalysis. Journal of Sports Sciences, 21, 443-457.

Zillman, D., \& Cantor, J. R., (1976). Effect of timing of information about mitigating circumstances on emotional response to provocation and retaliatory behavior. Journal of Experimental Social Psychology, 9, 282-293. 